\title{
Robust Power-Splitting SWIPT Beamforming for Broadcast Channels
}

\author{
Jialing Liao, Muhammad R. A. Khandaker, Member, IEEE, and Kai-Kit Wong, Fellow, IEEE
}

\begin{abstract}
This letter considers the multiple-input single-output (MISO) broadcast system for simultaneous wireless information and power transfer (SWIPT) using receiver power splitting and aims to optimize jointly the beamforming vectors and the power splitting ratios for minimizing the transmit power of the base station (BS) subject to the individual signal-to-interference-plusnoise ratio (SINR) and the energy-harvesting constraints at the mobile stations (MSs). However, the CSI is assumed imperfect but has a deterministic uncertainty region. Unlike existing attempts that resort to iterations guided by semidefinite relaxation (SDR), we propose a reverse convex nonsmooth optimization algorithm, which provides the near-optimal rank-one solution.
\end{abstract}

Index Terms-Energy harvesting, power splitting, optimal beamforming, broadcast, MISO.

\section{INTRODUCTION}

$\mathbf{T}$ HE CONTINUOUS efforts to tackle the challenge of battery-limited mobile communications have recently given rise to the concept of simultaneous wireless information and power transfer (SWIPT) and have since opened up numerous new opportunities. As an example, [1] characterized the rate-energy regions for multiple-input multiple-output (MIMO) broadcast systems for SWIPT with separated and colocated information and energy receivers. Other recent examples include the SWIPT work for orthogonal frequency-division multiplexing (OFDM) systems [2], frequency-selective channels [3] and multiuser scenarios, such as the interference channel, the relay channel [4], multicasting, and the broadcast channels [5].

In particular, the broadcast channel is a typical scenario of great interest, where the base station (BS) communicates with several mobile stations (MSs). Using SWIPT, each MS can be an information decoder (ID) as well as energy receiver (ER), either by time-switching or power splitting technologies.

Recently, the joint optimization problem of power splitting ratios and beamforming was studied in [5] for multiple-input single-output (MISO) SWIPT broadcast systems assuming perfect channel state information (CSI) at the BS. Later in [6], the results were extended to cope with the case of imperfect CSI, via a highly complex suboptimal two-step optimization process, which relies on alternatively solving semi-definite relaxation (SDR) problems with a $K$-dimensional search (where $K$ denotes the number of users in the broadcast system).

In this letter, we revisit the problem in [6] which aims to minimize the transmit power of the BS subject to the signal-tointerference and noise ratio (SINR) and the energy harvesting

Manuscript received August 28, 2015; accepted November 03, 2015. Date of publication November 9, 2015; date of current version January 7, 2016. This work was supported by EPSRC under Grant EP/K015893/1. The associate editor coordinating the review of this paper and approving it for publication was Z. Ding.

The authors are with the Department of Electronic and Electrical Engineering, University College London, London, U.K. (e-mail: jialing. liao.14@ucl.ac.uk; m.khandaker@ucl.ac.uk; kai-kit.wong@ucl.ac.uk).

Digital Object Identifier 10.1109/LCOMM.2015.2498928 constraints at the MSs, assuming the availability of imperfect CSI at the BS, for the MISO SWIPT broadcast system. ${ }^{1}$ The contributions of our proposed approach over [6] are twofold: (i) significant complexity reduction and (ii) near-optimality. In particular, we present a feasible SDR-guided randomization approach for the joint optimization of transmit beamforming and receive power splitting factors. On the contrary to [6], the SDRbased solution is non-iterative but only provides an upper-bound performance after rescaling. Hence, we propose a reverse convex constraint based penalty function method which guarantees a rank-one and near-optimal solution.

\section{System ModeL}

Consider a $K$-user MISO broadcast system as illustrated in Fig. 1, where the BS, with $N_{\mathrm{s}}$ antennas, communicates with $K$ single-antenna MSs. Each MS acts simultaneously as an ID and an ER via power splitting. With transmit beamforming at the BS, the received signal at the $k$ th MS can be written as

$$
y_{k}=\mathbf{h}_{k}^{H} \sum_{i=1}^{K} \mathbf{b}_{i} s_{i}+n_{\mathrm{A}, k}, \text { for } k=1, \ldots, K,
$$

where $\mathbf{b}_{i}$ and $s_{i}$ denote the transmit beamforming vector and the data symbol for the $i$ th MS, respectively, $\mathbf{h}_{k}$ is the channel vector between the $\mathrm{BS}$ and the $k$ th MS, $n_{\mathrm{A}, k}$ is the antenna noise at the $k$ th MS, and $(\cdot)^{H}$ is the Hermitian operation.

With a power splitter at the $k$ th MS, suppose that we have the power splitting ratio $\rho_{k} \in[0,1]$. Then the signal split to the ID of the $k$ th receiver is given by

$$
y_{\mathrm{I}, k}=\sqrt{\rho_{k}}\left(\mathbf{h}_{k}^{H} \sum_{k=1}^{K} \mathbf{b}_{i} s_{i}+n_{\mathrm{A}, k}\right)+n_{\mathrm{P}, k},
$$

where $n_{\mathrm{P}, k}$ denotes the additive noise at the ID of the $k$ th MS. Meanwhile, the signal split to the energy harvester of the $k$ th MS can be expressed as

$$
y_{\mathrm{E}, k}=\sqrt{1-\rho_{k}}\left(\mathbf{h}_{k}^{H} \sum_{i=1}^{K} \mathbf{b}_{i} s_{i}+n_{\mathrm{A}, k}\right) .
$$

As such, the SINR of the ID at the $k$ th MS is given by

$$
\operatorname{SINR}_{k}=\frac{\rho_{k} \mathbf{h}_{k}^{H} \mathbf{b}_{k} \mathbf{b}_{k}^{H} \mathbf{h}_{k}}{\rho_{k} \sigma_{\mathrm{A}, k}^{2}+\sigma_{\mathrm{P}, k}^{2}+\rho_{k} \mathbf{h}_{k}^{H}\left(\sum_{\substack{i=1 \\ i \neq k}}^{K} \mathbf{b}_{i} \mathbf{b}_{i}^{H}\right) \mathbf{h}_{k}},
$$

and the power harvested at the $k$ th MS is written as

$$
\mathrm{E}_{k}=\xi_{k}\left(1-\rho_{k}\right)\left(\mathbf{h}_{k}^{H}\left(\sum_{i=1}^{K} \mathbf{b}_{i} \mathbf{b}_{i}^{H}\right) \mathbf{h}_{k}+\sigma_{\mathrm{A}, k}^{2}\right),
$$

\footnotetext{
${ }^{1}$ For MIMO SWIPT broadcasting in [1], there is no rank-one issue and this means that existing results will not be optimal for MISO SWIPT systems.
} 


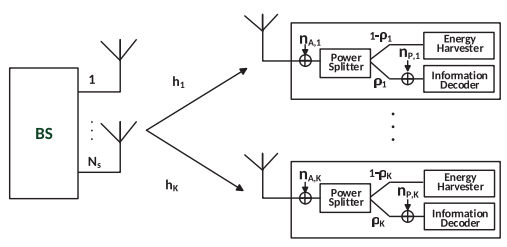

Fig. 1. A MISO SWIPT broadcast system with power splitters.

where $\xi_{k} \in(0,1]$ is the energy conversion efficiency of the energy harvester, and $\mathbb{E}\left\{\left|s_{i}\right|^{2}\right\}=1$ has been assumed.

\section{RoBUst OptIMIZATION}

In this letter, we model the channel by

$$
\mathbf{h}_{k}=\hat{\mathbf{h}}_{k}+\Delta \mathbf{h}_{k}, \quad \text { for } k=1, \ldots, K,
$$

where $\mathbf{h}_{k}$ is the actual channel vector, but $\hat{\mathbf{h}}_{k}$ denotes the CSI estimate with an error vector $\Delta \mathbf{h}_{k}$, which satisfies

$$
\left\|\Delta \mathbf{h}_{k}\right\|_{2}=\left\|\mathbf{h}_{k}-\hat{\mathbf{h}}_{k}\right\|_{2} \leq \varepsilon_{k}, \quad \text { for } \varepsilon_{k} \geq 0
$$

which reflects the quality of the estimates.

We aim to minimize the BS transmit power subject to the SINR and the energy-harvesting constraints at the MSs as

$$
\begin{aligned}
& \min _{\substack{\left\{\mathbf{b}_{k}\right\} \\
\left\{0<\rho_{k}<1\right\}}} \sum_{k=1}^{K} \mathbf{b}_{k}^{H} \mathbf{b}_{k} \text { s.t. } \\
& \min _{\Delta \mathbf{h}_{k}} \frac{\left|\mathbf{b}_{k}^{H}\left(\mathbf{h}_{k}+\Delta \mathbf{h}_{k}\right)\right|^{2}}{\gamma_{k}}-\sum_{\substack{i=1 \\
i \neq k}}^{K}\left|\mathbf{b}_{i}^{H}\left(\mathbf{h}_{k}+\Delta \mathbf{h}_{k}\right)\right|^{2} \\
& \geq \sigma_{\mathrm{A}, k}^{2}+\frac{\sigma_{\mathrm{P}, k}^{2}}{\rho_{k}}, \forall k, \\
& \min _{\Delta \mathbf{h}_{k}} \sum_{i=1}^{K}\left|\mathbf{b}_{i}^{H}\left(\mathbf{h}_{k}+\Delta \mathbf{h}_{k}\right)\right|^{2} \geq \frac{\eta_{k}}{\xi_{k}\left(1-\rho_{k}\right)}-\sigma_{\mathrm{A}, k}^{2}, \forall k,
\end{aligned}
$$

where $\gamma_{k}>0$ and $\eta_{k}>0$ are the given SINR and energy harvesting thresholds at the $k$ th MS, respectively. Due to imperfect CSI, however, our problem is not convex and has infinitely many constraints as opposed to that in [5].

Lemma 1 (S-Procedure): Let $f_{i}(\mathbf{x})=\mathbf{x}^{H} \mathbf{A}_{i} \mathbf{x}+2 \mathbf{b}_{i}^{H} \mathbf{x}+$ $c_{i}, i=1,2$ where $\mathbf{A}_{i} \in \mathbb{C}^{n \times n}, \mathbf{b}_{i} \in \mathbb{C}^{n}$ and $c_{i} \in \mathbb{R}$. The implication $f_{1}(\mathbf{x}) \leq 0 \Rightarrow f_{2}(\mathbf{x}) \leq 0$ holds if and only if there exists $\mu \geq 0$ satisfying

$$
\mu\left[\begin{array}{cc}
\mathbf{A}_{1} & \mathbf{b}_{1} \\
\mathbf{b}_{1}^{H} & c_{1}
\end{array}\right]-\left[\begin{array}{ll}
\mathbf{A}_{2} & \mathbf{b}_{2} \\
\mathbf{b}_{2}^{H} & c_{2}
\end{array}\right] \succeq \mathbf{0}
$$

Now, we define $\mathbf{W}_{k} \triangleq \mathbf{b}_{k} \mathbf{b}_{k}^{H}$ and substitute $\Delta \mathbf{h}_{k}^{H} \Delta \mathbf{h}_{k} \leq \varepsilon_{k}$ and (8b) into Lemma 1. We will then get a group of positive semidefinite matrices named $\left\{\boldsymbol{\Gamma}_{k}\right\}$ [see (10), shown at the bottom of the page] associated with parameters $\left\{\mu_{k}\right\}$. Similarly, if we apply S-Procedure to (8b) and $\Delta \mathbf{h}_{k}^{H} \Delta \mathbf{h}_{k} \leq \varepsilon_{k}$, then we will have another group of semi-definite matrices $\left\{\Upsilon_{k}\right\}$ [see (11), shown at the bottom of the page] associated with parameters $\left\{\lambda_{k}\right\}$.

Based on the S-Procedure, (8) becomes

$$
\begin{aligned}
\underset{\substack{\left\{\mathbf{W}_{k}\right\},\left\{\rho_{k}\right\} \\
\left\{\mu_{k}\right\},\left\{\lambda_{k}\right\}}}{ } \sum_{k=1}^{K} \operatorname{tr}\left(\mathbf{W}_{k}\right) \text { s.t. } \\
\boldsymbol{\Gamma}_{k}\left(\left\{\mathbf{W}_{k}\right\}, \rho_{k}, \mu_{k}\right) \succeq \mathbf{0}, \forall k, \\
\boldsymbol{\Upsilon}_{k}\left(\left\{\mathbf{W}_{k}\right\}, \rho_{k}, \lambda_{k}\right) \succeq \mathbf{0}, \forall k, \\
\mathbf{W}_{k} \succeq \mathbf{0}, 0<\rho_{k}<1, \forall k, \\
\mu_{k} \geq 0, \lambda_{k} \geq 0, \forall k, \\
\operatorname{Rank}\left(\mathbf{W}_{k}\right)=1, \forall k .
\end{aligned}
$$

Ignoring the rank-one constraint, problem (12) will be convex but cannot be solved by optimization packages CVX [7] due to the coupling of $\frac{1}{\rho_{k}}$ and $\frac{1}{1-\rho_{k}}$ in $\boldsymbol{\Gamma}_{k}$ and $\boldsymbol{\Upsilon}_{k}$. This was why [6] resorted to iterative suboptimal approaches. Here, we propose to solve the problem by introducing a group of new variables, $q_{k}$ and $\tilde{q}_{k}$ to get a definitely convex problem after rank relaxation which can be processed by existing solvers:

$$
\begin{array}{r}
\min _{\substack{\left\{\mathbf{W}_{k}\right\},\left\{\rho_{k}\right\},\left\{q_{k}\right\},\left\{\tilde{q}_{k}\right\},\left\{\mu_{k}\right\},\left\{\lambda_{k}\right\}}} \sum_{k=1}^{K} \operatorname{tr}\left(\mathbf{W}_{k}\right) \text { s.t. } \\
\tilde{\boldsymbol{\Gamma}}_{k}\left(\left\{\mathbf{W}_{k}\right\}, q_{k}, \mu_{k}\right) \succeq \mathbf{0}, \forall k, \\
\tilde{\boldsymbol{\Upsilon}}_{k}\left(\left\{\mathbf{W}_{k}\right\}, \tilde{q}_{k}, \lambda_{k}\right) \succeq \mathbf{0}, \forall k, \\
\mathbf{W}_{k} \geq \mathbf{0}, 0<\rho_{k}<1, \forall k, \\
q_{k} \geq \frac{1}{\rho_{k}}, \tilde{q}_{k} \geq \frac{1}{1-\rho_{k}}, \forall k, \\
\mu_{k} \geq 0, \lambda_{k} \geq 0, \forall k . \\
\operatorname{Rank}\left(\mathbf{W}_{k}\right)=1, \forall k .
\end{array}
$$

where $\tilde{\boldsymbol{\Gamma}}_{k}$ and $\tilde{\boldsymbol{\Upsilon}}_{k}$ are similar to those of $\boldsymbol{\Gamma}_{k}$ and $\boldsymbol{\Upsilon}_{k}$ except that we change $\frac{1}{\rho_{k}}$ and $\frac{1}{1-\rho_{k}}$ to $q_{k}$ and $\tilde{q}_{k}$, respectively.

Proposition 1: Regardless of the new variables $q_{k}$ and $\tilde{q}_{k}$, problems (12) and (13) are equivalent. The optimal solution to either of the two problems should also be optimal for the other one.

Proof: Please refer to Appendix A.

However, the rank-one constraint makes both (12) and (13) non-convex. To tackle this, SDR with randomization is used.

\section{A. SDR Guided Randomization}

In particular, the rank constraint is first dropped to obtain a suboptimal solution. Then the randomization technique is used

$$
\begin{aligned}
& \boldsymbol{\Gamma}_{k}\left(\left\{\mathbf{W}_{k}\right\}, \rho_{k}, \mu_{k}\right)=\left[\begin{array}{cc}
\mu_{k} \mathbf{I}_{N_{s}}+\frac{\mathbf{W}_{k}}{\gamma_{k}}-\sum_{\substack{i=1 \\
i \neq k}}^{K} \mathbf{W}_{i} & \frac{\mathbf{W}_{k} \mathbf{h}_{k}}{\gamma_{k}}-\sum_{\substack{i=1 \\
i \neq k}}^{K} \mathbf{W}_{i} \mathbf{h}_{k} \\
\frac{\mathbf{h}_{k}^{H} \mathbf{W}_{k}}{\gamma_{k}}-\sum_{\substack{i=1 \\
i \neq k}}^{K} \mathbf{h}_{k}^{H} \mathbf{W}_{i} & \frac{\mathbf{h}_{k}^{H} \mathbf{W}_{k} \mathbf{h}_{k}}{\gamma_{k}}-\sum_{\substack{i=1 \\
i \neq k}}^{K} \mathbf{h}_{k}^{H} \mathbf{W}_{i} \mathbf{h}_{k}-\sigma_{\mathrm{A}, k}^{2}-\frac{\sigma_{\mathrm{P}, k}^{2}}{\rho_{k}}-\mu_{k} \varepsilon_{k}
\end{array}\right] \succeq \mathbf{0}, \\
& \boldsymbol{\Upsilon}_{k}\left(\left\{\mathbf{W}_{k}\right\}, \rho_{k}, \lambda_{k}\right)=\left[\begin{array}{cc}
\lambda_{k} \mathbf{I}_{N_{s}}+\sum_{i=1}^{K} \mathbf{W}_{i} & \sum_{i=1}^{K} \mathbf{W}_{i} \mathbf{h}_{k} \\
\sum_{i=1}^{K} \mathbf{h}_{k}^{H} \mathbf{W}_{i} & \sum_{i=1}^{K} \mathbf{h}_{k}^{H} \mathbf{W}_{i} \mathbf{h}_{k}-\frac{\eta_{k}}{\xi_{k}\left(1-\rho_{k}\right)}+\sigma_{\mathrm{A}, k}^{2}-\lambda_{k} \varepsilon_{k}
\end{array}\right] \succeq \mathbf{0},
\end{aligned}
$$


to generate the feasible solutions to (12). Assuming that the solution of SDR is $\mathbf{W}_{k}^{*}, \forall k$, with the eigenvalue decomposition defined as $\mathbf{W}_{k}^{*}=\mathbf{U} \boldsymbol{\Sigma} \mathbf{U}^{H}$, the feasible beamforming vector of (12) under randomization can then be given by

$$
\mathbf{b}_{k}=\mathbf{U} \Sigma^{\frac{1}{2}} \mathbf{v}
$$

Here $\mathbf{U}$ is unitary and $\boldsymbol{\Sigma}$ is diagonal with eigenvalue arranged in decreasing order, and $\mathbf{v}$ is a vector of complex circularly symmetric uncorrelated Gaussian random variables with zero-mean and unit-variance. However, some of the constraints in (12) may be violated after randomization, and one needs to rescale the beamforming vector $\mathbf{b}_{k}$ with an appropriate factor $\alpha_{k}$ to meet the constraints. Thus we have

$$
\widetilde{\mathbf{b}}_{k}=\alpha_{k} \mathbf{b}_{k}, \forall k
$$

Then we reformulate the problem as follows and rely on CVX to derive the optimal scaling factors:

$$
\begin{gathered}
\min _{\left\{\beta_{k}\right\}} \sum_{k=1}^{K} \operatorname{tr}\left(\tilde{\mathbf{W}}_{k}\right) \text { s.t. } \\
\tilde{\boldsymbol{\Gamma}}_{k}\left(\left\{\tilde{\mathbf{W}}_{k}\right\}, q_{k}^{*}, \mu_{k}^{*}\right) \succeq \mathbf{0}, \forall k, \\
\tilde{\boldsymbol{\Upsilon}}_{k}\left(\left\{\tilde{\mathbf{W}}_{k}\right\}, \tilde{q}_{k}^{*}, \lambda_{k}^{*}\right) \succeq \mathbf{0}, \forall k, \\
\tilde{\mathbf{W}}_{k} \succeq \mathbf{0}, \forall k,
\end{gathered}
$$

where $\tilde{\mathbf{W}}_{k}=\beta_{k} \widetilde{\mathbf{b}}_{k} \widetilde{\mathbf{b}}_{k}^{H}, \beta_{k}=\alpha_{k}^{2}$, and $q_{k}^{*}, \mu_{k}^{*}, \tilde{q}_{k}^{*}, \lambda_{k}^{*}$ are the corresponding solution by the SDP approach. With the optimal scaling factors, we can easily generate $\mathbf{b}_{k}$ using (14) and (15).

The downside is that randomization always offers an upper bound solution due to the relaxation involved. As a remedy, in the following, we solve the problem by expressing the rank-one constraint $(13 \mathrm{~g})$ as a single reverse convex constraint which is then incorporated into the objective function as a penalty function. The resulting problem belongs to the class of concave programming with a nonsmooth objective.

\section{B. Penalty Function Method}

Since $\mathbf{W}_{k}, \forall k$ is always semi-positive definite, we then have $\operatorname{tr}\left(\mathbf{W}_{k}\right) \geq \lambda_{\max }\left(\mathbf{W}_{k}\right)$ where $\lambda_{\max }\left(\mathbf{W}_{k}\right)$ is the maximum eigenvalue of $\mathbf{W}_{k}$. In this case, if $\operatorname{tr}\left(\mathbf{W}_{k}\right) \leq \lambda_{\max }\left(\mathbf{W}_{k}\right)$ also holds, it will be easy to prove that $\operatorname{tr}\left(\mathbf{W}_{k}\right)=\lambda_{\max }\left(\mathbf{W}_{k}\right)$. That is to say, $\mathbf{W}_{k}$ has only one non-zero eigenvalue. Then we will have $\operatorname{Rank}\left(\mathbf{W}_{k}\right)=1, \forall k$. Thus, the rank-one constraints (13g) can be expressed by the single reverse convex constraint:

$$
\sum_{k=1}^{K}\left(\operatorname{tr}\left(\mathbf{W}_{k}\right)-\lambda_{\max }\left(\mathbf{W}_{k}\right)\right) \leq 0
$$

Note that the function $\lambda_{\max }(\mathbf{X})$ is convex on the set of Hermitian matrices. When $\operatorname{tr}\left(\mathbf{W}_{k}\right)-\lambda_{\max }\left(\mathbf{W}_{k}\right)$ is small enough, we will have $\mathbf{W}_{k} \approx \lambda_{\max }\left(\mathbf{W}_{k}\right) \mathbf{w}_{k, \max } \mathbf{w}_{k, \max }^{H}$, where $\mathbf{w}_{k, \text { max }}$ denotes the unit-norm eigenvector corresponding to the maximum eigenvalue $\lambda_{\max }\left(\mathbf{W}_{k}\right)$ (i.e., $\left.\left\|\mathbf{w}_{k, \max }\right\|=1\right)$. Then the optimal beamforming vector can be obtained as

$$
\mathbf{b}_{k}=\lambda_{\max }\left(\mathbf{W}_{k}\right)^{\frac{1}{2}} \mathbf{w}_{k, \max }
$$

satisfying the rank-one constraints $(13 \mathrm{~g})$. Our aim is therefore to make $\sum_{k=1}^{K}\left(\operatorname{tr}\left(\mathbf{W}_{k}\right)-\lambda_{\max }\left(\mathbf{W}_{k}\right)\right)$ as small as possible. Thus we consider the alternative formulation to (13):

$$
\min \tau \text { s.t. }
$$

$$
\begin{gathered}
\tilde{\boldsymbol{\Gamma}}_{k}\left(\left\{\mathbf{W}_{k}\right\}, q_{k}, \mu_{k}\right) \succeq \mathbf{0}, \forall k, \\
\tilde{\boldsymbol{\Upsilon}}_{k}\left(\left\{\mathbf{W}_{k}\right\}, \tilde{q}_{k}, \lambda_{k}\right) \succeq \mathbf{0}, \forall k, \\
\mathbf{W}_{k} \succeq \mathbf{0}, 0<\rho_{k}<1, \forall k, \\
q_{k} \geq \frac{1}{\rho_{k}}, \tilde{q}_{k} \geq \frac{1}{1-\rho_{k}}, \forall k, \\
\mu_{k} \geq 0, \lambda_{k} \geq 0, \kappa>0, \forall k .
\end{gathered}
$$

where $\tau \triangleq \sum_{k=1}^{K}\left(\operatorname{tr}\left(\mathbf{W}_{k}\right)+\kappa\left(\operatorname{tr}\left(\mathbf{W}_{k}\right)-\lambda_{\max }\left(\mathbf{W}_{k}\right)\right)\right)$ and $\kappa>$ 0 is a constant. If the weight $\kappa$ is chosen to be large enough, then the difference $\operatorname{tr}\left(\mathbf{W}_{k}\right)-\lambda_{\max }\left(\mathbf{W}_{k}\right)$ will be minimized. Clearly, the objective of (19) is to minimize both $\sum_{k=1}^{K} \operatorname{tr}\left(\mathbf{W}_{k}\right)$ and $\sum_{k=1}^{K}\left(\operatorname{tr}\left(\mathbf{W}_{k}\right)-\lambda_{\max }\left(\mathbf{W}_{k}\right)\right)$.

Lemma 2: Let $\mathbf{X}$ and $\mathbf{Y}$ be positive semidefinite matrices. Using the fact that a sub-gradient of $\lambda_{\max }(\mathbf{Y})$ is $\mathbf{y}_{\max } \mathbf{y}_{\max }^{H}$, we always have $\lambda_{\max }(\mathbf{X})-\lambda_{\max }(\mathbf{Y}) \geq \mathbf{y}_{\max }^{H}(\mathbf{X}-$ $\mathbf{Y}) \mathbf{y}_{\max }$. Meanwhile, $\lambda_{\max }(\mathbf{Y})$ and $\mathbf{y}_{\max }$ denote the maximum eigenvalue and corresponding eigenvector of $\mathbf{Y}$, respectively.

According to Lemma 2, given some feasible $\mathbf{W}_{k}^{(n)}$ of problem (19), we will have

$$
\begin{array}{r}
\operatorname{tr}\left(\mathbf{W}_{k}^{(n+1)}\right)+\kappa\left[\operatorname{tr}\left(\mathbf{W}_{k}^{(n+1)}\right)-\lambda_{\max }\left(\mathbf{W}_{k}^{(n)}\right)\right. \\
\left.-\left(\mathbf{W}_{k, \text { max }}^{(n)}\right)^{H}\left(\mathbf{W}_{k}^{(n+1)}-\mathbf{W}_{k}^{(n)}\right) \mathbf{W}_{k, \max }^{(n)}\right] \\
\leq \operatorname{tr}\left(\mathbf{W}_{k}^{(n)}\right)+\kappa\left(\operatorname{tr}\left(\mathbf{W}_{k}^{(n)}\right)-\lambda_{\max }\left(\mathbf{W}_{k}^{(n)}\right)\right),
\end{array}
$$

where the superscript $n$ denotes the $n$-th iteration. Accordingly, the following SDP problem gives an optimal solution $\mathbf{W}_{k}^{(n+1)}$ that is better than $\mathbf{W}_{k}^{(n)}$ of problem (19):

$$
\begin{aligned}
& \min _{\substack{\left\{\mathbf{W}_{k}\right\},\left\{\rho_{k}\right\},\left\{q_{k}\right\} \\
\left\{\tilde{q}_{k}\right\},\left\{\mu_{k}\right\},\left\{\lambda_{k}\right\}}} \sum_{k=1}^{K} \operatorname{tr}\left(\mathbf{W}_{k}^{(n+1)}\right)+\kappa\left[\operatorname{tr}\left(\mathbf{W}_{k}^{(n+1)}\right)-\lambda \max \left(\mathbf{W}_{k}^{(n)}\right)\right. \\
& \left.\quad-\left(\mathbf{w}_{k, \max }^{(n)}\right)^{H}\left(\mathbf{W}_{k}^{(n+1)}-\mathbf{W}_{k}^{(n)}\right) \mathbf{w}_{k, \max }^{(n)}\right] \\
& \text { s.t. (19b)-(19f). }
\end{aligned}
$$

Now, (21) can be further simplified to

$$
\begin{gathered}
\min _{\substack{\left\{\mathbf{W}_{k}\right\},\left\{\rho_{k}\right\},\left\{q_{k}\right\} \\
\left\{\tilde{q_{k}}\right\},\left\{\mu_{k}\right\},\left\{\lambda_{k}\right\}}} \sum_{k=1}^{K} \operatorname{tr}\left(\mathbf{W}_{k}\right)+\kappa\left[\operatorname{tr}\left(\mathbf{W}_{k}\right)-\left(\mathbf{w}_{k, \max }^{(n)}\right)^{H} \mathbf{W}_{k}\right. \\
\left.\quad \times \mathbf{w}_{k, \max }^{(n)}\right] \\
\text { s.t. (19b)-(19f). }
\end{gathered}
$$

Due to the initial condition $\operatorname{tr}\left(\mathbf{W}_{k}^{(0)}\right)-\lambda_{\max }\left(\mathbf{W}_{k}^{(0)}\right)=0$, at some $n$, we will have $\operatorname{tr}\left(\mathbf{W}_{k}^{(n)}\right)-\lambda_{\max }\left(\mathbf{W}_{k}^{(n)}\right)=0$.

The proposed nonsmooth iterative algorithm to resolve the rank-one beamforming problem is summarized in Algorithm 1.

\section{Simulation Results}

In this section, the performance of the proposed methods is investigated via simulations. We considered $\gamma_{k}=\gamma, \eta_{k}=\eta$, $\xi_{k}=0.5, \sigma_{\mathrm{A}, k}^{2}=10^{-8}$, and $\sigma_{\mathrm{P}, k}^{2}=10^{-6}, \varepsilon_{k}=0.001, \forall k$. The channel vector is modeled as

$$
\mathbf{h}_{k}=\frac{1}{\sqrt{d_{k}^{m_{k}}}}\left(\sqrt{\frac{K_{R}}{1+K_{R}}} \mathbf{h}_{k}^{\mathrm{LOS}}+\sqrt{\frac{1}{1+K_{R}}} \mathbf{h}_{k}^{\mathrm{NLOS}}\right),
$$



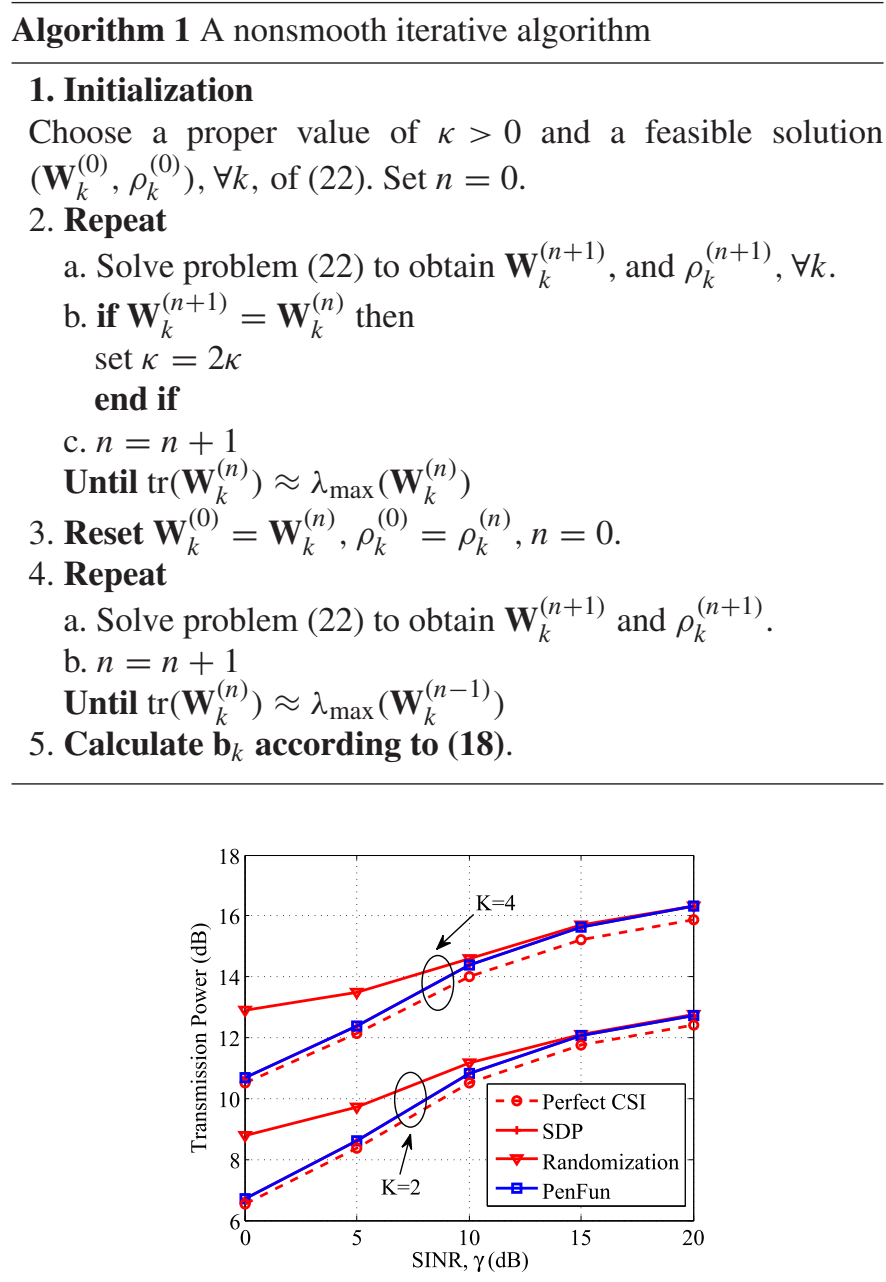

Fig. 2. The BS transmit power versus the $\operatorname{SINR} \gamma$.

where $\mathbf{h}_{k}^{\mathrm{LOS}}=10^{-2}\left[1, e^{j \theta_{k}}, e^{j 2 \theta_{k}}, \ldots, e^{j\left(N_{s}-1\right) \theta_{k}}\right]^{T}$ with $\theta_{k}=$ $-\pi \sin \phi_{k}, \phi_{k} \in[-\pi, \pi]$ is randomly generated and the Rician ratio $K_{R}=5 \mathrm{~dB}, d_{k}(=1.5)$ and $m_{k}(=2.7)$ denote the BS to MS distances and the path loss exponents, respectively, with reference to [4], and $\mathbf{h}_{k}^{\mathrm{NLOS}}$ is an independent zero-mean complex Gaussian random variable with variance of $10^{-2}$.

Fig. 2 shows the performance in terms of the transmit power versus SINR targets $(\gamma)$ and fixed harvested power threshold $\eta=10 \mathrm{dBm}$ with $K=2$ and $K=4$, respectively. Here we set $N_{s}=4$. The randomization approach, the penalty function method (PenFun), the SDP method, and the optimal performance with perfect CSI [5] are all compared. As can be observed, the minimum transmit power rises with the increase of the number of MSs. Also in both cases, the randomization approach shows an upper-bound performance compared with the other methods due to randomization. PenFun also performs nearly as the SDP method and is also quite close to the perfect CSI case which demonstrates that the proposed PenFun method not only guarantees a rank-one solution but also yields the global optimal solution. Moreover, the gap between PenFun and the randomization approach is narrowed when increasing the SINR threshold while that between the PenFun method and the perfect CSI case follows a reverse trend.

Next, we compare the performance of the methods mentioned above versus the energy harvesting threshold $\eta$ with targeted SINR fixed at $\gamma=10 \mathrm{~dB}, K=4$ and $N_{s}=4$ or 8 in Fig. 3.

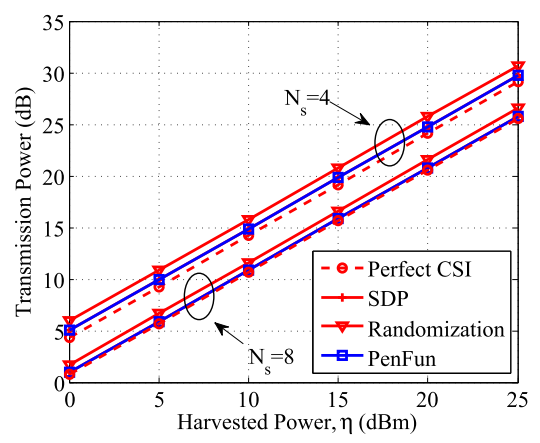

Fig. 3. Transmission power versus harvested power $\eta$.

Similarly, in this figure the PenFun method outperforms the randomization approach and shows comparable performance to the SDP method, and the perfect CSI case. Also, increasing the number of antennas at BS can reduce the minimum demanded transmit power to some degree.

\section{Conclusions}

In this letter, a MISO SWIPT broadcast system was investigated. The joint-optimal transmit beamforming and powersplitting ratio with imperfect CSI was obtained using penalty function method. In particular, we have shown that the penalty function method yields a more reliable and better solution.

\section{APPENDIX A \\ PROOF OF PROPOSITION 1}

Suppose $\left(\mathbf{W}_{k}^{*}, \rho_{k}^{*}, \mu_{k}^{*}, \lambda_{k}^{*}\right)$ be the optimal solution of (12). Letting $q_{k}^{*}=\frac{1}{\rho_{k}^{*}}$, and $\tilde{q}_{k}^{*}=\frac{1}{1-\rho_{k}^{*}}$, it is easy to see that $\left(\mathbf{W}_{k}^{*}, \rho_{k}^{*}, q_{k}^{*}, \tilde{q}_{k}^{*}, \mu_{k}^{*}, \lambda_{k}^{*}\right)$ also satisfies the constraints in (13). Oppositely, if $\left(\mathbf{W}_{k}^{*}, \rho_{k}^{*}, q_{k}^{*}, \tilde{q}_{k}^{*}, \mu_{k}^{*}, \lambda_{k}^{*}\right)$ is the optimal solution for (13), then $\boldsymbol{\Gamma}_{k}$ and $\boldsymbol{\Upsilon}_{k}$ will both be positive semi-definite (PSD) due to the fact that $\boldsymbol{\Gamma}_{k}-\tilde{\boldsymbol{\Gamma}}_{k} \succeq \mathbf{0}, \boldsymbol{\Upsilon}_{k}-\tilde{\boldsymbol{\Upsilon}}_{k} \succeq \mathbf{0}$. Also, the objective function is not directly related to $q_{k}^{*}, \tilde{q}_{k}^{*}$ such that we can solve (13) with CVX instead of (12).

\section{REFERENCES}

[1] R. Zhang and C. K. Ho, "MIMO broadcasting for simultaneous wireless information and power transfer," IEEE Trans. Wireless Commun., vol. 12, no. 5, pp. 1989-2001, May 2013.

[2] X. Zhou, R. Zhang, and C. K. Ho, "Wireless information and power transfer: Architecture design and rate-energy tradeoff," IEEE Trans. Commun., vol. 61, no. 11, pp. 4754-4767, Nov. 2013.

[3] L. Liu, R. Zhang, and K. C. Chua, "Wireless information transfer with opportunistic energy harvesting," IEEE Trans. Wireless Commun., vol. 12, no. 1, pp. 288-300, Jan. 2013.

[4] A. Nasir, X. Zhou, S. Durrani, and R. Kennedy, "Relaying protocols for wireless energy harvesting and information processing," IEEE Trans. Wireless Commun., vol. 12, no. 7, pp. 3622-3636, Jul. 2013.

[5] Q. Shi, L. Liu, W. Xu, and R. Zhang, "Joint transmit beamforming and receive power splitting for MISO SWIPT systems," IEEE Trans. Wireless Commun., vol. 13, no. 6, pp. 3269-3280, Jun. 2014.

[6] Z. Zhu, Z. Wang, X. Gui, and X. Gao, "Robust downlink beamforming and power splitting design in multiuser MISO SWIPT system," in Proc. IEEE/CIC Int. Conf. Commun. China, Oct. 2014, pp. 271-275.

[7] M. Grant and S. Boyd. (2013, Sep.). CVX: Matlab Software for Disciplined Convex Programming, Version 2.0 Beta [Online]. Available: http://cvxr.com/cvx

[8] A. H. Phan, H. D Tuan, H. H. Kha, and D. T. Ngo, "Nonsmooth optimization for efficient beamforming in cognitive radio multicast transmission," IEEE Trans. Signal Process., vol. 60, no. 6, pp. 2941-2951, Jun. 2012. 